\title{
Correlation of HbA1c with Serum Lipid Profile in Patients with Type 2 Diabetes Mellitus
}

\author{
Dr.Satarupa Dash \\ Assistant Professor, DeptPhysiology, VIMSAR,Burla,Odisha
}

\begin{abstract}
Background: Diabetes mellitus is a heterogeneous group of disease, characterized by a state of chronic hyperglycemia, resulting from a diversity of etiologies, genetic and environmental factors acting jointly.Glycated heamoglobin $(\mathrm{HbAlc})$ is considered a gold-standard measure of chronic glycaemia in diabetic patients. HbAlc is a main indicator for mean blood glucose level. Dyslipidemia especially high LDL, is common in diabetes mellitus and strongly associated with poor glycaemia control.

Objective-To find the correlation of HbAlC with lipid profile in patients with Type 2 Diabetes Mellitus.

Material \&Method: A total of 60 confirmed cases of type 2 diabetes mellitus were obtained from VIMSAR Medicine Department. The samples comprised both males and females. ( $40-60$ YR).60 subjects without dibetes of similar age group were taken as control. The parameters FBS, PPBS, HbAlc, TG, Chol, HDL, LDL, VLDL were studied and compared between case and control group.

Observation: $F B S, P P B S, H b A I C$ were significantly high in case group. $L D L, C H O L, T G$ were high in case as compared to control group, HDL is significantly low compared to control group. HbAlC /LDL, HbAlC /HDL, HbAlC /CHOL statistically strongly significant results in patients when compared to controls.

Result- HbAlc is significantly correlated with lipid profiles.

Conclusion: Type 2 diabetic patients are more prone to dyslipidaemia. HbAlc is significantly correlated with lipid profiles. So HbAlc can be used as an indirect indicator of dyslipidaemia in type 2 diabetes in addition to as glycemic control biomarker.
\end{abstract}

Keywards:Type 2 diabetes mellitus, HbAlc, lipid profile

\section{Introduction}

Diabetes mellitus is a heterogeneous group of disease, characterized by a state of chronic hyperglycemia, resulting from a diversity of etiologies, genetic and environmental factors acting jointly. The underlying cause of diabetes is the impaired production or action of insulin, a hormone that controls glucose, fat and amino acids metabolism. The metabolic dysregulations associated with diabetes mellitus (DM) causes secondary pathophysiological changes in multiple organ systems that impose a tremendous burden on the individual with diabetes and on health care system. Two broad categories of diabetes are designated as type 1 and type 2 diabetes mellitus (DM). Type 2 DM is more common than type 1 DM. India is called the diabetic capital of the world. Type 2 diabetes mellitus in Indians is being increasingly seen in younger and less obese persons than in western countries. Most of the longstanding macro and micro vascular complications are also more common among Indian diabetics as compared to other races and ethnic groups. Recent studies have shown that the prevalence of coronary heart disease (CHD) in Indian diabetics may be as high as in the migrant population. [1] Changes in lipid-profile are a consequential event in DM. Due to these changes distribution and function of various fractions of lipids are affected. Many Studies have evaluated the risk factors for CHD in DM patients and observed high fasting blood sugar (FBS) and post prandial blood sugar (PPBS), total cholesterol (Chol), low density lipoproteins (LDL), triglycerides (TG) levels and low high density lipoproteins (HDL) levels when compared to controls. [2]

Glycated heamoglobin (HbA1c) is considered a gold-standard measure of chronic glycemia in diabetic patients. It was studied that HbAlc was a better CHD predictor than fasting or 2-h glucose.[3]. HbAlc was strongly associated with atherosclerosis as measured by carotid IMT (intima-media thickness). [4,5] .The ADA recommends measurement of $\mathrm{HbAlc}$ in patients with both type 1 and 2 diabetes, first to document the degree of glycemic control, then as part of continuing care.[6].Changes in lipid profile is also well related with severity of DM as adjudged by HbA1c.

Dyslipidemia (raised triglycerides, raised cholesterol, raised LDL, raised VLDL and low HDL) were common in type 2 diabetes mellitus, which is further associated with insulin resistance like hyperinsulinemia, hypertension with obesity collectively known as metabolic syndrome or Reaves's syndrome; strongly correlated with atherosclerosis. Atherogenic indices i.e. TC/HDL-C ratio $(<5)$ and LDL-C/HDL-C $(<3.5)$ is the main indicator of cardiovascular disease (CVD). TG/HDL-C which is considered as reliable as fasting serum insulin levels and is determined to assess insulin resistance status in type 2 diabetes. [7].Dyslipidemia, especially high 
LDL, is common in diabetes mellitus and strongly associated with poor glycemic control. Glycated hemoglobin (HbA1c) is main routinely used tool for measuring long term glycemiccontrol .[8] . HbA1c is a main indicator for mean blood glucose level; HbA1c predicts the risk of diabetic complications in diabetic patients. Thus, in the present study an attempt has been made to find any correlation of HbA1c with lipid profile in patients with type 2 diabetes mellitus.

\section{2.a Study participants}

\section{Material Method}

A total of 60 confirmed cases of type 2 diabetes mellitus were obtained from VIMSAR Medicine Department Unit IV. The samples comprised both males and females. All diabetic patients were aged $40-60$ Years. The informed consent form was voluntarily taken from each diabetic patient who participated in the study.60 subjects without diabetes were taken as control. The parameters studied were FBS, PPBS, HbA1c, TG, Chol, HDL, LDL, VLDL in both case and control group. This study was approved by institutional ethical committee.

2.b Measurement of Biochemical Parameters After an overnight fasting, approximately $5 \mathrm{ml}$ venous blood was collected with the help of disposable syringe. The serum was separated immediately after collecting the blood sample via centrifugation at $3000 \mathrm{rpm}$ for 10 minutes. Fasting blood glucose concentrations were measured within 30 minutes to 1 hour of sample collection. Lipid profile (total cholesterol, triglycerides, high density lipoprotein) was measured using automatic blood analyzercobas c 111(according to the manufacturer's instructions). Low density lipoprotein and very low density lipoproteins were measured using the Friedewald's formula (given below)

Low density lipoprotein $=$ Total Cholesterol $-($ High density lipoprotein - Triglycerides $/ 5)$ VLDL= Triglycerides $/ 5$

The Glycated hemoglobin (HbA1c) were measured with a radioimmunoassay (HbA1c LD-500 Analyzer)

2.c Statistical Analysis Standard descriptive statistics (mean \pm standard deviation) were determined for directly measured variable. Unpaired t test were applied to establish the correlations of anthropometric variables, FBG, Hba1c, Lipid profiles, atherogenic indices . Data were analyses using SPSS (Statistical Package for Social Science) version 20.0. A p $<0.05$ was used to indicate statistical significance.

\section{Observation}

Table 1 shows the demographic distribution between patient and control group . Samples age, gender $\&$ BMI are matched with $\mathrm{P}<0.05$. Table 2 gives the results of FBS, PPBS \& HbA1c levels of patients and control group presented as Mean $\pm \mathrm{SD}$. There is increase in all 3 parameters in patients compared to controls which is statistically strongly significant $(\mathrm{p}<0.05)$. Table 3 gives the results of lipid profile levels of patients and control group expressed as Mean \pm SD. There is strongly significant increase in TG, LDL \& cholesterol levels in patients compared to controls, whereas HDL showed statistical decrease. No significant change in VLDL of case compared to control. Table 4 gives the results of association between HDL, LDL, Chol and with HbA1c (HbA1c / HDL, HbA1c / LDL, HbA1c / Chol) levels of patients and control group. HbA1c / HDL, HbA1c / LDL \& HbA1c / Chol ratios shows statistically strongly significant results in patients when compared to controls. The results of the study parameters FBS, PPBS, HbA1c, TG, Chol, HDL, LDL, VLDL are depicted in tabular form.

\section{Discussion}

The present study shows FBS \& PPBS values of patients higher than upper limit which correlated well with the clinical diagnosis. HbA1c is done to monitor the control of blood glucose in diabetes mellitus. Several studies have shown the positive correlation of $\mathrm{HbA1c}$ with duration of DM and as a strong predictor of risk (cardiovascular diseases) for diabetes complications. [9]. HDL, LDL, TG and Chol are well known risk factors for complications of DM like Coronary heart disease. In a study by H. Surekha Rani Et.al., it is observed that FBS and PPBS, Chol, VLDL, LDLs, TGs were high and the levels of HDLs were low compared to controls. [10].In diabetic dyslipidemia, high TG tend to coexist with low HDL and small, dense, undesirable (more atherogenic) type of LDL in their blood (even though their LDL level may be normal). In the present study we found significantly increased levels of TG and VLDL and decreased HDL levels. Study by Elizabeth et.al, observed that LDL and HDL cholesterol were significantly associated with HbA1c. HDL cholesterol was inversely associated with HbA1c where as LDL cholesterol was positively associated with HbA1c in diagnosed diabetics. [11].In present study there is a significant difference in the HbA1c/LDL \& HbA1c/Chol ratios between cases and controls. Similarly we also observed a significant difference in HbA1c/HDL ratio $(\mathrm{p}<0.05)$ indicating the inverse relation between HDL and the HbAlc levels. This is similar to study done by MahanteshPatil et al.[12]. 
Lipid abnormalities are common in diabetic patients and frequently seen in patients with type-2 diabetic mellitus. The abnormal lipid profile observed in type 2 Diabetes mellitus is said to be related to insulin resistance as reported in previous studies, which leads to increased release of free fatty acids from fatty tissue, impaired insulin dependent muscle uptake of free fatty acids and increase fatty acid release to the hepatic tissue. [13]. It has been closely associated with diabetic dyslipedemia, hypertension and enormous risk to cardiovascular diseases.[14]. Chronic hyperglycemia causes glycation of apolipoproteins and interferes with the normal pathways of lipoprotein metabolism. [15]. Diabetic patients have much complication which includes elevated levels of LDL and triacylglycerol and low level of HDL. In the present study, the results showed that the lipid profile were higher in diabetic patients and that they were in agreement with the finding of Wexler et al., 2005 .[16]. In the present study there is highly positive significant correction were observed between HbA1c and Lipid profiles (TC, TG and LDL). HbA1c has significant positive correlation ( $\mathrm{p}<0.016-0.001)$ with FBG, TC, TG, LDL-C and LDL-C/HDL-C.[17].Erciyas et al, (2004) also reported the positive correlation between HbA1c and TC, TG; in patients with type-2 diabetes .[18].The Diabetic complication and control trial (DCCT) established HbA1c as the gold standard of glycemic control. The HbAlc value $<7.0 \%$ reduced the risk of cardiovascular diseases and value $>7.0 \%$ leads to dyslipedemia to the patients. [19]. Controlling the glycemic levels may significantly decrease the risk of cardiovascular diseases in diabetes. Khawetal has reported that reducing the HbA1c level by $0.2 \%$ could lower the mortality by $10 \%$ thus the present study suggests the importance of glycemic control in prevention of cardiovascular diseases in type 2 diabetes. [20].

\section{Conclusion}

It was concluded from the results of this study that type 2 diabetic patients are more prone to dyslipidaemia. HbA1c is significantly correlated with lipid profiles. So HbA1c can be used as an indirect indicator of dyslipidaemia in type 2 diabetes in addition to as glycemic control biomarker. Thus early diagnosis of dyslipidaemia can be used as a preventive measure for development of cardiovascular disease (CVD) in patients with type 2 diabetes mellitus. Patients should be educated about regular monitoring of lipid profiles and if found to be abnormal, should control blood glucose and cholesterol very effectively.

Tables-

\begin{tabular}{|l|l|l|l|}
\hline & CASES & CONTROL & P value \\
\hline AGE & $50.10 \pm 12.86$ & $49.05 \pm 9.66$ & $<0.05$ \\
\hline MALE & 38 & 36 & $<0.05$ \\
\hline FEMALE & 22 & 24 & $<0.05$ \\
\hline TOTAL & 60 & 60 & \\
\hline H/O DM( IN YEARS) & $5 \pm 1.7$ & NIL & \\
\hline BMI & $22.38 \pm 4.2$ & $25.1 \pm 1.83$ & $<0.05$ \\
\hline
\end{tabular}

Table 2

\begin{tabular}{|l|l|l|l|}
\hline Parameters & Case & control & P VALUE \\
\hline FBS & $200.03 \pm 49.67$ & $96.20 \pm 8.28$ & $<0.05$ \\
\hline PPBS & $259.50 \pm 50.39$ & $108.20 \pm 19.2$ & $<0.05$ \\
\hline HbA1C & $7.73 \pm 0.76$ & $5.11 \pm 0.27$ & $<0.05$ \\
\hline
\end{tabular}

Table 3

\begin{tabular}{|l|l|l|l|}
\hline LIPID PARAMETER & CASE & CONTROL & P VALUE \\
\hline TOTAL CHOLESTERL & $177.07 \pm 9.92$ & $169.20 \pm 4.57$ & $<0.05$ \\
\hline TRIGLYSERIDE & $156.10 \pm 9.16$ & $120.60 \pm 8.25$ & $<0.05$ \\
\hline LDL & $135.63 \pm 9.57$ & $100.85 \pm 2.11$ & $<0.05$ \\
\hline VLDL & $42.20 \pm 6.07$ & $40.80 \pm 4.9$ & $>0.05$ \\
\hline HDL & $42.47 \pm 6.08$ & $46.05 \pm 6.64$ & $<0.05$ \\
\hline
\end{tabular}

Table 4

\begin{tabular}{|l|l|l|l|}
\hline PARAMETERS & CASE & CONTROL & P VALUE \\
\hline $\mathrm{HbA1C/CHOL}$ & $0.07 \pm 0.08$ & $0.29 \pm 0.01$ & $\mathrm{t}=6.8, \mathrm{p}<0.05$ \\
\hline $\mathrm{HbA} 1 \mathrm{C} / \mathrm{HDL}$ & $0.28 \pm 0.04$ & $0.15 \pm 0.03$ & $\mathrm{t}=5.5, \mathrm{p}<0.05$ \\
\hline $\mathrm{HbA} 1 \mathrm{C} / \mathrm{LDL}$ & $0.10 \pm 0.07$ & $0.05 \pm 0.04$ & $\mathrm{t}=8.7, \mathrm{p}<0.05$ \\
\hline
\end{tabular}




\section{Reference}

[1]. Lt Gen SR Mehta, VSM, Col AS Kashyap, Lt Col S Das. Diabetes Mellitus in India: The Modern Scourge. MJAFI 2009;65:50-4

[2]. Surekha Rani. H, Madhavi G, RamachandraRaoV,Sahay B.K, Jyothy A. Risk Factors for Coronary Heart Disease in Type II DM. Indian Journal of Clinical Biochemistry 2005;20(2):75-80.

[3]. Zachary T,Bloomgarden. Cardiovascular Disease, Neuropathy and Retinopathy.Diabetes care 2009;32: e64- e 68.

[4]. Chambless LE, Heiss G, Folsom AR, Rosamond W,Szklo M, Sharrett AR, Clegg LX. Association of coronary heart disease incidence with carotid arterial wall thickness and major risk factors: the Atherosclerosis MahanteshPatilet. al. Int J Cur Res Rev, March 2014/ Vol 06 (06) Page 24 Risk in Communities (ARIC) Study, 1987- 1993. Am J Epidemiol 1997;146:483-94.

[5]. Burtis Carl A, Ashwood Edward R, Bruns David E.Tietz Textbook of clinical chemistry and molecular diagnostics. 4th Ed. New dehli: Elsevier publishers; 2008. Chapter 26,Lipids, lipopropteins, apolipoproteins and other cardiovascular risk factors: p. 903-83.

[6]. American Diabetes Association. Standards of medical care for patients with diabetes mellitus. Diabetes Care 2000;23(Suppl 1):S32-42. 10

[7]. Rath M, Ncendorf A, Reblin T, Dietel M, Knebber HJ. Detection and quantification of lipoprotein(a) in the arterial wall of 107 coronary bypass patients. Atherosclerosis 1989; 9:579-92.

[8]. KhareKC, Raman PG, Bhatnagar AD, ReemaBhavsar. Serum Lp(a) levels in patients of diabetes mellitus. Int. J. Diab. Dev. Countries 2000;20:79-83

[9]. Robert McCarter J, James Hempe M, Ricardo Gomez, Stuart Chalew A. Biological Variation in HbA1c Predicts Risk of Retinopathy and Nephropathy in Type 1 Diabetes. Diabetes Care 2004;27:1259-64.

[10]. Surekha Rani. H, Madhavi G, RamachandraRaoV,Sahay B.K, Jyothy A. Risk Factors for Coronary Heart Disease in Type II DM. Indian Journal of Clinical Biochemistry 2005;20(2):75-80.

[11]. Elizabeth Selvin, Josef Coresh, Sherita Golden H, Lori L. Boland,FrederickBrancati L, Michael Steffes W. Glycemic Control, Atherosclerosis, and Risk Factors for Cardiovascular Disease in Individuals with Diabetes. Diabetes Care. 2005;28(8):1965-73.

[12]. MahanteshPatil, Nirmal Kumar, Aliya Nusrath, Shubha Jayaram, Rajeshwari A. ASSOCIATION OF HbA1c WITH SERUM LIPID PROFILE AND LIPOPROTEIN (a) IN TYPE 2 DIABETES MELLITUS Int J Cur Res Rev; $2014 ; 06$ (06) ; 20 -25.

[13]. Boden G; Role of fatty acids in the pathogenesis of insulin resistance and NIDDM. Diabetes, 1997; $46(1): 4755$.

[14]. Mgonda YM, Ramaiya KL, Swai AB, McLarty DG, Alberti KG; Insulin resistance and hypertension in non obese Africans in Tanzania. Hypertension, 1998; 31(1):114-118.

[15]. Carmena R; High risk of lipoprotein dysfunction in type 2 diabetes mellitus. Rev EspCardiolSupl., 2008; 8 (supl C): 18C-24C.

[16]. Wexler, D.J., Grant, R.W., Meigs, J.B., Nathan, D.M and Cagliero, E. Sex disparities in the treatment of cardiac risk factors in patients with type 2 diabetes. Diabetes Care, 2005; 28:514-520.

[17]. Rosmee and ShyamalKoley . Association of HbA1c with Lipid profiles in patients with type 2 diabetes mellitus . International Journal of Biomedical Research 2016; 7(3): 139-143.

[18]. Erciyas, F., Taneli, F., Arslan, B., and Uslu, Y. Glycemic control, oxidative stress and lipid profile in children with type 1 Diabetes Mellitus. Arch. Med. Res. 2004; 35:134-140.

[19]. Rohlfling, C.L., Wiedmeyer, H.M., Little, R.R., England, J.D., Tennill, A. and Goldstein, D.E. Defining the relationship between plasma glucose and HbA1c: analysis of glucose profiles and HbA1c in Diabetes Control and Com-plications trial. Diabetes Care 2002; 25: 275-278

[20]. Khaw, K.T., Wareham, N., Luben, R., Bingham, S., Okas, S., Welch, A., Glycated hemoglobin, diabetes, and mortality in men in Norfolk cohort of European Prospective Investigation of Cancer and Nutrition (EPICNorfolk). Br. Med. J. 2001; 322:15-18. 